\title{
Mercadeo interno en las pequeñas y medianas empresas del sector salud de Barranquilla (Colombia)
}

\author{
Karen M. Payares ${ }^{(1) \star}$, Margel A. Parra ${ }^{(2)}$, Evaristo J. Navarro(3) y Oscar Naranjo(4) \\ (1) Universidad de la Costa, Departamento de Ciencias Empresariales, Barranquilla, Colombia. \\ (e-mail: kpayares4@cuc.edu.co). \\ (2) Universidad de la Costa, Departamento de Ciencias Sociales, Barranquilla, Colombia. (e-mail: mparra3@cuc.edu.co) \\ (3) Universidad de la Costa, Departamento de Ciencias Naturales y Exactas, Barranquilla, Colombia. \\ (e-mail: enavarro3@cuc.edu.co) \\ (4) Institución Universitaria CEIPA, Docente, Barranquilla, Colombia. (e-mail: oscar.naranjo@ceipa.edu.co)
}

* Autor a quien debe ser dirigida la correspondencia.

Recibido Mar. 21, 2019; Aceptado May. 10, 2019; Versión final Jun. 26, 2019, Publicado Feb. 2020

\section{Resumen}

Se analiza el mercadeo interno (marketing interno) en las pequeñas y medianas empresas del sector salud de Barranquilla, con un enfoque metodológico deductivo, paradigma cuantitativo y diseño no experimental de campo. Como técnica se usa la encuesta, utilizando un cuestionario de escala tipo Likert, aplicado a 16 personas del área de servicio al cliente de la empresa sujeto de estudio. Los datos recolectados fueron procesados a través del análisis factorial, identificándose que la empresa entendida como el producto representa la carga factorial más alta con una participación del $89 \%$. Se concluye que en la aplicación de estrategias de marketing interno en el sector salud, la estabilidad laboral, el sistema de recompensas y las herramientas de comunicación influyen positivamente para tener un recurso humano motivado, satisfecho y fidelizado. Por tal motivo, el mantener vínculos organizacionales sólidos en la actualidad, constituye un reto para los líderes de las compañías.

\section{Internal marketing in small and medium size companies in the health service sector of Barranquilla (Colombia)}

\begin{abstract}
The internal marketing in small and medium-sized companies of the health sector in Barranquilla is analyzed, using a deductive methodological approach, a quantitative paradigm and a non-experimental field design. A survey is used as technique for collecting information, using a scale Likert type questionnaire, which applied to 16 people belonging to the customer service of the company being studied. The collected data were processed through factor analysis, identifying that the company understood as the product represents the the highest factorial load with $89 \%$. It is concluded that in the application of internal marketing in the health sector strategies, employment stability, system of rewards, and communication tools, have a positive influence to achieve a motivated, satisfied and loyal workforce. For this reason, maintaining strong organizational links today is a challenge for company leaders.
\end{abstract}




\section{INTRODUCCIÓN}

En el marco del entorno dinámico y competitivo de las organizaciones, las empresas buscan ser eficientes, optimizar su calidad, lograr posicionamiento y reconocimiento, incluso establecer una cultura empresarial que le permita generar ventajas competitivas en el mercado global, es así como los altos dirigentes concentran sus esfuerzos en el desarrollo de estrategias que puedan responder de forma efectiva a las variaciones del entorno donde opere la compañía en el que intervienen competidores, cambios económicos, avances tecnológicos, incluso ventajas competitivas que influyen en el éxito de la empresa (Barra, 2017). En relación a las estrategias organizacionales, estas deben plantearse y ejecutarse soportadas en los distintos recursos materiales, tecnológicos, financieros y humanos que posea la empresa, teniendo en cuenta que estos últimos se constituyen en el activo intangible más valioso de la organización y por consiguiente en un factor que permite la consecución de los logros empresariales se considera importante mantener la motivación del colaborador (Payares et al. 2017). Una de las estrategias que interviene en la estimulación del empleado es el marketing interno, el cual reconoce al colaborador como un cliente interno que se debe mantener satisfecho y para ello se necesita hacerlos participes de la generación de cambios y toma de decisiones de la compañía, mediante el suministro de información precisa a través de los canales de comunicación establecidos al interior de la empresa (Berry, 1981; Mendoza et al., 2011).

Las estrategias de marketing al interior de la empresa deben estar orientadas a la motivación del empleado y al bienestar de este con fines de entregar más que una transacción comercial, una oferta de valor al cliente externo, para llevar esta estrategia a cabo es necesario promocionar una compañía con objetivos claros, dirigentes reconocidos y una estructura administrativa identificable, que le permita al empleado sentirse parte de la organización. Kotler y Keller (2006) sustentan que todas las actividades encauzadas a la fidelización del empleado y satisfacción de sus necesidades repercuten en el cumplimiento de las metas organizacionales, para ello es indispensable reclutar y capacitar a individuos con habilidades para ofrecer un servicio de atención al cliente óptimo, debido a que solo se puede ofrecer excelente servicio cuando se está preparado para suministrarlo.

Es así como el marketing interno se define como la gestión que se realiza en compañía del marketing externo y con fines de satisfacer al cliente externo, por ende es un elemento interdisciplinario que merece estudio en el ámbito empresarial, en razón a que sus aportes son de gran relevancia y contribuyen en el primer ámbito, al desarrollo del conocimiento y en el segundo al desarrollo de estrategias de gestión empresarial que se traduzcan en rentabilidad para la organización; la estrategia de marketing interno debe ser una actividad cautelosa y equilibrada, es decir que su funcionamiento estará acorde con los recursos financieros, de esta manera se podrá desarrollar una efectiva gestión del cliente interno y sus resultados positivos afectaran en la rentabilidad y productividad de la empresa (Bohnemberger et al. 2019).

En un contexto dinámico donde las barreras geográficas propenden a desaparecer y las brechas culturales enmarcan parámetros de nuevos mercados, los cuales deben procurar potencializar sus recursos, con la finalidad de promover el desarrollo social y productivo, las pequeñas y medianas empresas, (Pymes) se consideran como unidades productivas que se caracterizan por cumplir con esta premisa, respondiendo a exigencias del entorno, superando sus expectativas y manteniendo el estatus de agentes económicos que tienen mayor impacto en la economía de Latinoamérica (Castillo et al., 2017). La Organización para la Cooperación y el Desarrollo Económicos -OCDE- (2017) afirma que las Pymes constituyen de forma ascendente el $90 \%$ del total de las compañías en América Latina, siendo responsables de un $80 \%$ de participación en la generación de empleo, impactando significativo en la economía. Para Acopi (2017) en el territorio colombiano las Pymes están orientadas a mantener una alta competitividad en el mercado donde se desarrollan, ya que por su tamaño tienen una ventaja de adaptación a los requerimientos del medio y una capacidad de concentración en nichos de mercado muy específicos, que les permitirá mantenerse al nivel de la competencia, las Pymes representan el 95\% del conglomerado empresarial del país y de este porcentaje el $46 \%$ despliega sus actividades en el sector servicios.

Del sector servicios la fracción correspondiente a la salud en el departamento del Atlántico se enmarca en el 6.81\%, ubicándolo así en el tercer pilar del índice de competitividad del departamento (Fundesarrollo, 2017). Así mismo resultados de investigaciones hechas por la Cámara de Comercio de Barranquilla y Fundesarrollo (2015) indica que el Clúster Salud-Farma, conformado por empresas e instituciones cuya actividad económica principal es la producción, distribución y consumo de bienes y servicios que protejan y promuevan la salud de las personas, determina entre las actividades principales, aquellas que están orientadas al servicio de la salud a través de clínicas especializadas, hospitales, centros de diagnóstico entre otros. Los servicios de la salud es uno de los sectores con mayor sensibilidad y exigencias en cuanto a la identificación de su personal y prestación de un servicio de calidad, se hace necesario desarrollar estrategias que permitan el desarrollo de ventajas competitivas y sostenibles en el mercado. Teniendo en cuenta el porcentaje de la participación de las Pymes, la contribución que estás realizan al sector servicios, específicamente las instituciones prestadoras de salud (IPS), definidas según el ministerio de salud de Colombia como aquellas instituciones que tienen como función prestar los servicios de salud en el nivel de 
atención que se requiera tanto a los afiliados como beneficiarios cobijados por las leyes en las que se contemplen (Minsalud, 2019) específicamente del departamento del Atlántico, así como también la importancia de su ubicación estratégica en los índices de competitividad del departamento y la necesidad de su desarrollo permanente, se presenta el objetivo del estudio que consiste en analizar el marketing interno en las pequeñas y medianas empresas del sector salud (IPS) de Barranquilla - Atlántico.

\section{OTROS ANTECEDENTES}

Reconocer al empleado como un cliente dentro de las organizaciones, genera colaboradores satisfechos, por ende se hace necesario la inclusión y cooperación de estos, dado que la participación puede generar cambios en la disposición de los empleados y redundar así de forma positiva en los clientes externos (Berry, 1981). El colaborador debe suponerse como un cliente interno, el cual debe someterse a procesos de selección capacitación y motivación en aras de fomentar sentido de pertenencia en el mismo, es así como el mercadeo interno rediseña el rol del empleado (Johnston ,1989). De acuerdo a las anteriores premisas, adoptando estas estrategias en la organización se generan trasformaciones con respecto a la motivación, bienestar y compromiso con los empleados, entendiendo que estos son la base que permite concebir ventajas competitivas en el mercado, así mismo son participantes activos de los procesos rutinarios donde el objetivo es la entrega de servicio que satisface al cliente externo (Prieto et al., 2016).

Es así como se reafirma que la satisfacción del cliente externo se logra a través de los empleados, generando una motivación y concientización en los colaboradores sobre la importancia del consumidor final dado que indistintamente de los niveles jerárquico de la organización, todos influyen en la calidad del servicio que se brinda es así como las relaciones y comportamiento organizacional son sustanciales dentro de la compañía (Gronroos, 1990). El marketing interno es una actividad de relacionamiento que apoya a la gerencia y tiene como fin desarrollar en el equipo lineamientos de comunicación interna que propicien ventajas en el proceso organizacional, en razón que permite concientizar al empleado sobre la importancia de la calidad de la información que se maneje en la empresa. (Salehzadeh y Jamkhaneh, 2019; Araque et al., 2017; López et al., 2016 y Dantas y Soares, 2016).

Al respecto es necesario precisar que el marketing interno es entendido como la estrategia orientada a satisfacer al empleado y los resultados de su implementación recaen en la calidad de los servicios ofrecidos por la empresa, un empleado satisfecho ofrece un excelente servicio al cliente externo, de allí que los colaboradores se constituyen en un primer mercado competitivo en donde se debe procurar que los altos mandos se preocupen por equiparar sus necesidades, generar una red de motivación y cuidad su entorno económico y social (Vieira y Gonçalves, 2018; Morais y Soares, 2016). El objetivo del marketing interno además de enfocarse en que el trabajador se sienta identificado o que desarrolle un sentido de pertenecía con la organización, también está enfocado en la importancia del aporte que pueda realizar el trabajador al logro de los objetivos empresariales, al implementarse se obtendría un mayor beneficio para la organización (Azêdo y Alves, 2013; Jungsun y Choong, 2016). Bang et al. (2017); Rodrigues et al. (2016) y Fortenberry y McGoldrick, (2016) afirman que el marketing interno se contempla como una decisión que hace parte de la estrategia organizacional cuya finalidad es trabajar primordialmente en la motivación de los empleados quienes son el recurso más valioso que tiene la empresa y los que permiten agregar valor a la misma. Las estrategias orientadas al interior de la organización deben propiciar relaciones sólidas en relación con el empleado tomando como premisa que los factores comunicacionales y motivacionales permiten elevar el compromiso de los colaboradores hacia la organización.

En este sentido el marketing interno es una estrategia que debe anteceder al marketing externo, debido a que solo se puede ofrecer un buen servicio cuando se está dispuesto a suministrarlo. Así mismo, se considera que las organizaciones utilizan los llamados componentes de la mezcla de mercadeo para responder a las necesidades del mercado, entendiendo que todas las acciones ejecutadas lograran una estimulación sobre la demanda de sus productos y servicios los cuales se disponen a través del producto, precio, plaza y promoción (Kotler y Keller 2006). En efecto es imperante que las compañías establezcan relaciones consistentes con sus consumidores, garantizando ventajas competitivas sólidas, por consiguiente es necesario propiciar un ambiente organizacional donde el objetivo sea el bienestar del empleado y que las acciones ejecutadas redunden en la atracción y fidelización de clientes externos, en razón que la rentabilidad de la compañía se encuentra relacionada con las prácticas de marketing interno aplicadas (Bansal et al., 2001) y (Balemba y Bugandwa, 2016).

Para llevar a cabo las estrategias de marketing interno se ejecuta un proceso de tres etapas, en primera instancia se debe recopilar información con las oportunidades de mejora de la organización de acuerdo a los segmentos que se encuentren en la misma, seguido comunicar los hallazgos encontrados a fin de analizarlos, es importante resaltar que la comunicación bidireccional entre los altos mandos y empleados generan impacto positivo en la compañía; por último la estrategia debe finalizar generando soluciones y respuestas que correspondan a las necesidades identificadas (Robledo y Vallespín 2014). Por otra parte Papasolomou y Vrontis (2006) y Fang et al. (2014) aportan cuatro ejes centrales del marketing interno: 
capacitación y entrenamiento, sistema de recompensas y salarios, calidad de los equipos que se le suministren a la hora de desarrollar las actividades al empleado, así como su área de trabajo y por supuesto el cliente interno. Estos temas equivalen a las 4P difundidas en el ámbito académico y organizacional. Así mismo el autor establece que implementando los ejes mencionados se construyen paradigmas que permiten una cultura organizacional orientada al cliente interno, redundando en el servicio al consumidor final.

No obstante a la reseña anterior los elementos contenidos en la mezcla de marketing interno son la forma de generar las estrategias donde se debe desarrollar relaciones de calidad entre la empresa y sus empleados teniendo en cuenta como principal facilitador la comunicación y motivación, pues son éstos los que generan un trabajo mancomunado y en equipo que permite elevar el compromiso y sentido de pertenencia a los colaboradores por ende es primordial para la compañía orientarlos hacia el logro de los objetivos misionales a través de la difusión de los lineamientos filosóficos empresariales que se encuentran alineadas a los objetivos de la misma. Los elementos son: producto, objetivo, despliegue y comunicación interno entendiéndoles como:(a) Producto=Producto: comprende el diseño del programa de marketing en función a la satisfacción del cliente interno y con orientación a la satisfacción del cliente externo. (b)Precio = Objetivo: Respuesta esperada del empleado luego de emplear las estrategias del marketing interno, es decir si se desarrolló un programa con finalidades de entrenamiento y capacitación, el colaborador debería generar valor a la empresa, ser más productivo. (c) Plaza =Despliegue: se refiere a los elementos que utiliza la empresa para ejecutar las acciones de un plan de marketing interno, diseñar las etapas para que un programa se lleve a cabo y se obtenga respuesta positiva del empleado. (d) Promoción = Comunicación Interna: los recursos y actividades empleadas en función de la comunicación de los objetivos del programa de marketing interno a desarrollar en los empleados se utilizan videos, carteles, multimedia, correos electrónicos entre otros (Caridad et al. 2017).

Por su parte Gronroos (1990) expone una estructura en la que la gestión en función del empleado tiene un protagonismo sustancial con el fin de darle importancia al mismo y mantener su motivación. La información de las campañas publicitarias es importante hacérselas saber al cliente interno, este modelo se caracteriza por proponer dos alternativas, la primera consiste en mayor interacción para lograr un aumento en las ventas y la segunda obedece a la calidad de servicio y satisfacción del cliente. Sin embargo, no menciona mecanismos de motivación para el empleado, pero si sustenta que los elementos del marketing mix interno permiten el logro de ventajas competitivas. (a) Cliente Interno: Trabajador al cual se le aplica las estrategias de marketing interno. (b)Producto: Es la compañía que tiene como filosofía la interacción del empleado con las actividades de la organización. (c) Objetivo: Calidad del servicio percibida, cliente externo satisfecho, por ende, aumento en las ventas y la rentabilidad que debe ser el resultante de la aplicación de las estrategias de marketing interno. (d) Promoción= Comunicación: Mantener actualizado al trabajador con la información de la empresa por ejemplo de campañas publicitarias antes de salir al cliente externo. (e)Motivación: El empleado debe sentirse como parte fundamental de la organización, de esta forma se propenderá por la motivación.

Para Mohamed y Pervaiz (2000) al empleado se le debe reconocer como un cliente interno el cual se debe mantener satisfecho para propender por la calidad de los servicios ofrecidos por la empresa, y para que esta actividad se lleve a cabo con éxito, los trabajadores deben ser partícipes de las actividades que realice la empresa, promoviendo la satisfacción en el ámbito laboral de tal manera que se generara satisfacción en el consumidor final. Referente a los elementos el autor propone sus definiciones de la siguiente manera: (a) Producto: tareas a realizar asignadas por la organización teniendo en cuenta los propósitos misionales de ésta. (b)Empleado: Cliente Interno al que se le aplica estrategias de marketing interno. (c)Comunicación Interna: Comunicar al empleado las estrategias del marketing interno. (d)Precio: Aplicación interna de técnicas del marketing. (e)Objetivo: Satisfacción del cliente externo, lograr ventajas competitivas.

Abordando las presunciones teóricas del marketing interno desde sus elementos se construye conceptos sobre los mismos considerando solo los predominantes y comunes entre los autores representativos, los cuales son producto, precio, plaza y promoción, a continuación sus definiciones en la tabla 1.

Tabla 1: Definición de los Elementos de Marketing Interno

\begin{tabular}{|l|l|}
\hline Elementos & Definición \\
\hline Producto & Estabilidad laboral, beneficios, compensaciones y bienestar que la empresa le ofrece al empleado. \\
\hline Precio & $\begin{array}{l}\text { Políticas salariales que establece la organización y las cuales deben definirse de acuerdo a la } \\
\text { experiencia laboral y formación profesional del empleado. }\end{array}$ \\
\hline Plaza & $\begin{array}{l}\text { Recursos, lugar y mecanismos que la empresa suministra al empleado para que este ejecute sus } \\
\text { funciones de manera óptima. }\end{array}$ \\
\hline Promoción & $\begin{array}{l}\text { Estrategias del proceso comunicacional que se desarrollan al interior de la empresa donde } \\
\text { intervienen todos los niveles de mando que convergen en la estructura organizacional. }\end{array}$ \\
\hline
\end{tabular}




\section{METODOLOGÍA}

El desarrollo metodológico se constituye en el enfoque empírico analítico toda vez que permite medir una variable de estudio de forma objetiva a través de datos confiables, respecto al tipo de investigación del presente artículo se considera de tipo aplicado cuantitativo (Hernández et al., 2014). Con un diseño no experimental en razón que no se manipulara la variable ni se alterara el contexto debido a que se estudiaran los hechos tal como se presentan en la realidad y de esta forma se analizaran los sucesos en un contexto natural. Así mismo la investigación que origina el artículo realizado fue de campo, considerando los postulados teóricos de Arias (2012) quien afirma que este tipo de investigación es aquella que recolecte datos de la realidad donde ocurren los sucesos o de los individuos directamente relacionados, siendo estos datos primarios.

Hernández et al. (2014) expresa que los estudios de corte deductivo, inician con teoría y de ésta se esgrima juicios designados como hipótesis los cuales el investigador tiene como objetivo poner a prueba; es decir, la investigación parte de unas premisas preconcebidas, para luego medir las variables objeto de estudio mediante el diseño que se ha preestablecido, pues solo de ésta manera podría mantener la rigurosidad y el control de la investigación. En ese sentido en la presente investigación se utilizó el método deductivo, en razón que las variables se estudiaran a partir de fenómenos generales hasta llegar a un suceso particular en una situación determinada debido a que se trabajó sobre los conceptos generales del marketing interno en el contexto de las pequeñas y medianas empresas del sector salud (IPS) de Barranquilla. Así mismo se precisa que la población tenida en cuenta ha sido finita en razón que son de pleno conocimiento las unidades que integran a la misma.

En coherencia a los objetivos planteados, para efectos de la población se determinó las dieciocho (18) pequeñas y medianas empresas del sector salud de barranquilla (IPS), suministradas por la base de datos emitida por la cámara de comercio de Barranquilla. En la población objeto de estudio fungirán como unidades informantes los empleados quienes cumplieron con los siguientes criterios: se encontraban en el cargo de atención al cliente, con más de un (1) año laborando en la empresa, contratación directa y en un rango de edades de 22 a 45 años. A quienes se le aplico un instrumento diseñado con escala tipo Likert, el cual fue sometido a revisión por expertos para constatar la validez del mismo. Con respecto a la muestra se empleó el muestreo no probabilístico intencional, es preciso que, de la población, solo dieciséis (16) empresas tuvieron la intención de participar. El muestreo no probabilístico intencional se define como el método de selección en el cual no se conoce la probabilidad de que las unidades informantes hagan parte de la muestra, para efectos de esta investigación fueron uno por cada institución es decir (16) dieciséis, a su vez establece que los elementos son seleccionados en coherencia a los criterios del investigador, para efectos de esta investigación se consideraron los siguientes criterios, 1) que fueran pequeñas y medianas empresas, 2) Sector salud, 3) IPS (Arias, 2012).

\section{RESULTADOS}

El sector salud demanda que sus empleados desarrollen sus actividades motivados, fidelizados y alineados a los objetivos organizacionales con la finalidad de estar en capacidad de suministrar servicios de calidad y aumentar su competitividad, en este sentido se presentan los datos arrojados producto de la aplicación del instrumento de medición el cual tuvo como objetivo analizar el marketing interno en las pequeñas y medianas empresas del sector salud (IPS) de Barranquilla, teniendo en cuenta la operacionalización de las variables de estudio y las bases teóricas de Kotler y Keller (2006). De acuerdo al análisis factorial realizado, a partir del coeficiente $r$ de Pearson donde se identificó el grado de relación existente entre las dimensiones del marketing interno se logró evidenciar que existen correlaciones tanto contrarias como positivas entre las dimensiones de plaza, producto, precio y promoción de las pymes del sector salud de Barranquilla, tal y como se demuestra en la tabla 2.

Tabla 2: Matriz de correlaciones entre las dimensiones del marketing interno. a. Determinante $=, 0011$

\begin{tabular}{|l|l|c|c|c|c|}
\hline \multicolumn{2}{|c}{} & Plaza & Producto & Precio & Promoción \\
\hline \multirow{4}{*}{ Correlación } & Plaza & 1 & $-0,386$ & 0,03 & $-0,016$ \\
\cline { 2 - 6 } & Producto & $-0,386$ & 1 & 0,697 & 0,513 \\
\cline { 2 - 6 } & Precio & 0,03 & 0,697 & 1 & 0,614 \\
\cline { 2 - 6 } & Promoción & $-0,016$ & 0,513 & 0,614 & 1 \\
\hline \multirow{4}{*}{ Sig. (Unilateral) } & Plaza & & 0,07 & 0,456 & 0,477 \\
\cline { 2 - 7 } & Producto & 0,07 & & 0,001 & 0,021 \\
\cline { 2 - 7 } & Precio & 0,456 & 0,001 & & 0,006 \\
\cline { 2 - 6 } & Promoción & 0,477 & 0,021 & 0,006 & \\
\hline
\end{tabular}


En lo que respecta al elemento plaza se evidencia correlaciones positivas con respecto a los precios representados en un $30 \%$ y contrarios con las dimensiones producto $-38 \%$ y promoción $-16 \%$, lo que reafirma los postulados de Papasolomou y Vrontis (2006) refiriéndose a la importancia del sistema de recompensas en la compañía. En lo concerniente al producto se evidencian correlaciones positivas con precio en $69 \%$ y promoción $51 \%$ sin embargo la plaza demuestra denota una correlación negativa en -38\%, lo que respalda a Mohamed y Pervaiz (2000) quienes sostienen que la organización entendida como el producto y la promoción como la comunicación son elementos fundamentales para desarrollar hacia el cliente interno. Para el precio las correlaciones apuntan positivamente en dirección a la plaza con $30 \%$, producto ascendiendo al $69 \%$ y promoción con $61 \%$ lo que se respalda en los postulados de Papasolomou y Vrontis (2006) quien resalta la importancia del precio para el cliente interno. Para la dimensión promoción las correlaciones fueron positivas en dirección al producto con $51 \%$ y precio con $61 \%$, aun así, para la plaza arrojo un resultado negativo de $-16 \%$ lo que respalda las presunciones de López et al. (2016) referido a la herramienta de comunicación al interior de la empresa. Posterior al análisis de las relaciones entre las dimensiones del marketing interno donde se demuestra la existencia de interrelaciones propias de la variable objeto de estudio, se implementa la prueba de esfericidad de Barlett y el análisis Káiser, Meyer y Olkin (KMO), mediante el software estadístico SPSS, esta medida de adecuación central revela que la muestra de los datos en su totalidad es apropiada, arrojando los resultados evidenciados en la tabla 3.

Tabla 3: KMO y prueba de Barlett

\begin{tabular}{|c|c|r|}
\hline \multicolumn{2}{|c|}{ Medida de adecuación muestral de Káiser-Meyer-Olkin. } &, 74 \\
4 \\
\hline \multirow{2}{*}{ Prueba de esfericidad de Bartlett } & Chi-cuadrado aproximado & 19, \\
\cline { 2 - 3 } & Gl & 649 \\
\cline { 2 - 3 } & Gl & \\
\hline
\end{tabular}

De acuerdo a los resultados de la medida de la adecuación muestral de Káiser-Meyer-Olkin y la prueba de esfericidad de Barlett se comprueba la viabilidad de realizar un análisis factorial que permita conocer el porcentaje de dependencia entre las dimensiones del marketing interno, para efectos de este estudio se implementara un análisis de componentes principales y de acuerdo a la tabla 4 se demuestra que el componente 1 representa el $56.421 \%$ de la variación sobre el marketing interno en las pequeñas y medianas empresas del sector salud de Barranquilla, teniendo en cuenta lo anterior expresado será el componente producto de análisis.

Tabla 4: Varianza total explicada. Método de extracción: Análisis de Componentes principales

\begin{tabular}{|c|c|c|c|c|c|}
\hline \multirow{2}{*}{ Componente } & \multicolumn{3}{|c|}{ Autovalores iniciales } & \multicolumn{2}{c|}{$\begin{array}{c}\text { Sumas de las saturaciones al } \\
\text { cuadrado de la extracción }\end{array}$} \\
\cline { 2 - 6 } & Total & $\%$ de la varianza & $\%$ acumulado & Total & \% de la varianza \\
\hline 1 & 2,257 & 56,421 & 56,421 & 2,257 & 56,421 \\
\hline 2 & 1,105 & 27,63 & 84,051 & 1,105 & 27,63 \\
\hline 3 & 0,45 & 11,238 & 95,289 & & \\
\hline 4 & 0,188 & 4,711 & 100 & & \\
\hline
\end{tabular}

Así mismo, se evidencia en la tabla 5 el análisis factorial del marketing interno en el entorno de las pequeñas y medianas empresas del sector salud de Barranquilla, donde el elemento producto que hace referencia a la empresa representa la carga factorial más alta con un $89 \%$, continuando con el precio que es entendido como la remuneración con $87 \%$, seguido de la promoción que se traduce en las políticas de comunicación con $79 \%$ y finalmente la plaza con una participación negativa del $26 \%$ sobre el total de la variable, es así como los resultados se respaldan en las presunciones de Gronroos (1990) quien expuso que las actividades encaminadas al empleado relacionadas con el crecimiento y la interacción del mismo hacen parte de la filosofía de la compañía por ende son inherentes a la ejecución de la misión organizacional y que el precio se constituye en un factor importante siendo el equivalente de los costos y políticas de remuneración, así como también el conjunto de enseñanzas y prácticas de la importancia de la funciones de los empleados al interior de la compañía. Los hallazgos demuestran que en función a las 
actividades de difusión en la empresa, los colaboradores deben ser los primeros en ser informados sobre los cambios y toma de decisiones consultadas por medio de participación activa y métodos para la buena comunicación de esta manera se fortalece la interacción en los equipo de trabajo de la compañía (Araque et al., 2017; López et al., 2016; Salehzadeh y Jamkhaneh, 2019). Finalmente, en lo concerniente al elemento plaza, es imperativo generar un espacio donde se desarrolle la gestión participativa con fines únicos de la satisfacción, importancia y motivación del cliente interno dentro de la organización (Kotler y Keller, 2006).

Tabla 5: Matriz de componentes. Método de extracción: Análisis de componentes principales

\begin{tabular}{|c|c|}
\hline Dimensiones & Componente1 \\
\hline Producto & 0,890 \\
\hline Precio & 0,875 \\
\hline Promoción & 0,794 \\
\hline Plaza & $-0,263$ \\
\hline
\end{tabular}

\section{DISCUSIÓN FINAL}

Los resultados evidencian en primer lugar que existe presencia de los elementos plaza, precio, producto y promoción en el contexto estudiado. A través del análisis factorial implementado se determina que dentro de las dimensiones que componen el marketing interno tiene mayor importancia el producto, entendido como la estabilidad laboral y los beneficios que les puede generar la compañía tanto a nivel organizacional como personal a los empleados; lo que representa un reto para los dirigentes de las instituciones prestadoras de salud sugiriéndoles que se ejecuten acciones en donde se haga explicito las estrategias que la empresa implementa en función del cliente interno permitiendo fortalecer vínculos organizacionales que redunden en el cumplimiento de los objetivos misionales de la empresa. Entre las distintas estrategias que permitirían el fortalecimiento del sector salud se pueden distinguir las siguientes:

Entendiendo el elemento precio como las políticas de remuneración que tiene la empresa y contemplado como la inversión que se realiza en capacitaciones y entrenamientos dirigidos al empleado se concibe que tiene una participación relevante dentro de los elementos que componen el marketing interno, por lo que establecer políticas de remuneración de forma justa y acorde a las labores realizadas en donde el empleado se le otorgue su salario teniendo en cuenta no solo su profesión, sino el aporte en conocimiento que puede realizar a través de sus capacidades desarrolladas dentro el sector salud, de tal manera que permita enriquecer los procesos que lleve a cabo las ips de este tipo, desarrollan en el colaborador un vínculo organizacional, que los líderes de las pymes (IPS) deben considerar como aspecto fundamental en su actividad gerencial.

En lo concerniente a estrategias de comunicación y considerando la importancia de esta dimensión para la eficiencia en entidades de servicio como el sector salud, se sugiere que en el proceso comunicacional se posibiliten espacios como reuniones periódicas donde la retroalimentación y la intervención de participantes de los distintos niveles jerárquicos sea una premisa fundamental, dado que bajo este mecanismo se promueve el fortalecimiento de las acciones concernientes a la disposición de las IPS para contribuir a la agilidad, eficiencia y capacidad de respuesta hacia las necesidades del empleado con fines de brindar soluciones que el colaborador se sienta satisfecho y por ende motivado a brindar una excelente experiencia de servicio al cliente final. Comprendiendo el aporte que hacen las IPS contenidas en las pequeñas y medianas empresas del sector salud en el desarrollo económico del departamento del Atlántico y su contribución en el PIB del país, así como también su proyección dentro del clúster de Salud-Farma, a partir de las estrategias antes descritas se generarían oportunidades encausadas al fortalecimiento del servicio, satisfacción y motivación del empleado lo que generaría ineludiblemente el ofrecimiento de un servicio de calidad al cliente final - usuario.

De acuerdo a los resultados obtenidos en la presente investigación y teniendo en cuenta las teorías disciplinares de Berry (1981) y Gronroos (1990), se ha diseñado un modelo de marketing interno que define los cuatro elementos base que componen la variable de estudio. Estos, al ser adoptados intrínsecamente en la organización generan un empleado satisfecho y motivado que propende por el cumplimiento de los objetivos misionales, fortaleciendo vínculos con la compañía y desarrollando sentido de pertenencia a la misma. La premisa permite que el colaborador ofrezca una excelente atención al cliente externo que redunda en una experiencia de servicio diferenciador que conlleva a establecer relaciones comerciales sólidas con el consumidor final para finalmente obtener como resultado un aumento en la rentabilidad de la empresa, en búsqueda de la competitividad e innovación. La figura 1 describe el modelo de marketing. 


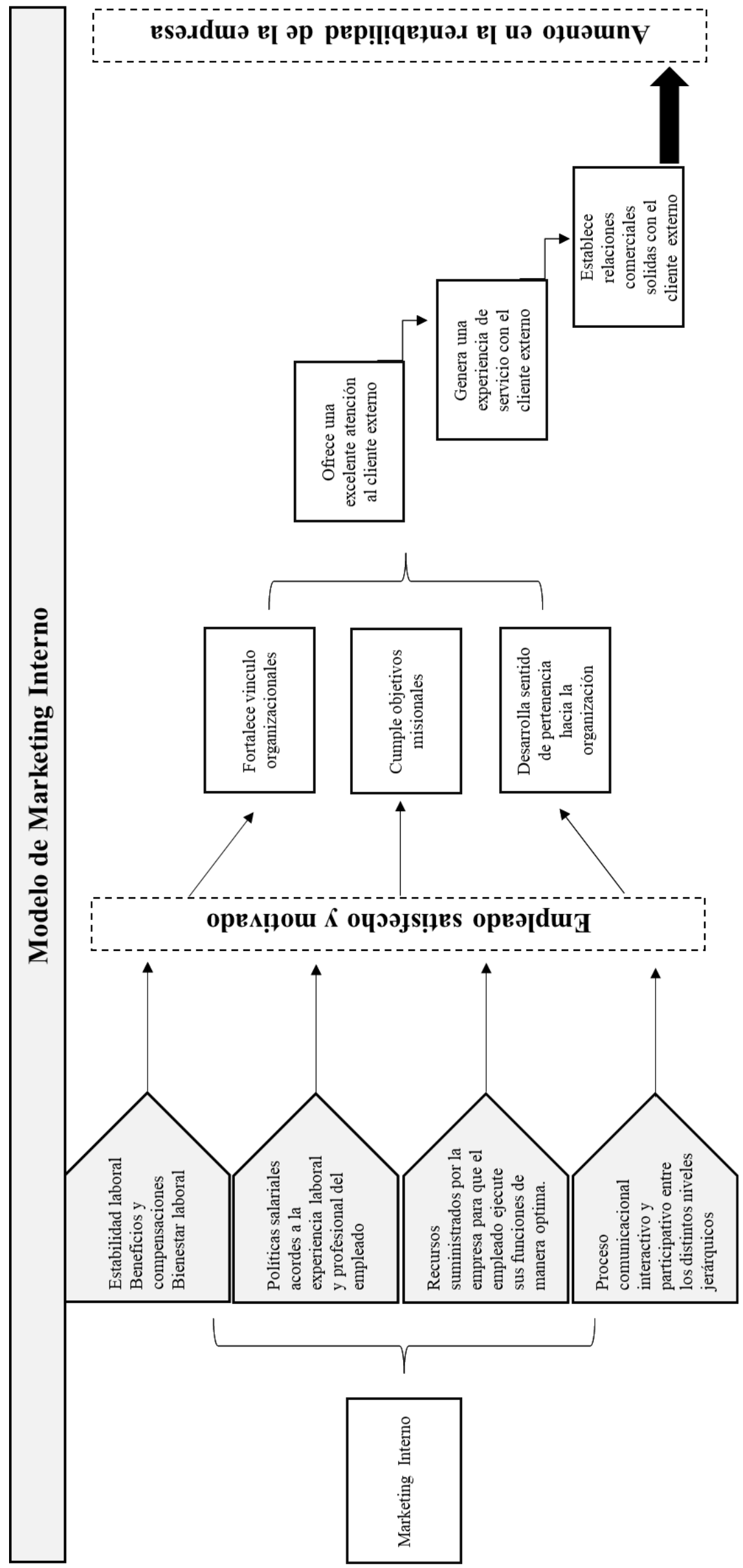

Fig. 1: Modelo de marketing interno 


\section{CONCLUSIONES}

A partir de la investigación realizada la cual tuvo como objetivo analizar los elementos del marketing interno en las pequeñas y medianas empresas del sector salud de Barranquilla se concluye: (1) evidencian los elementos: producto, precio, plaza y promoción, donde prima el producto, concebido en el contexto de estudio como los beneficios laborales, bienestar y permanencia que se le otorga al empleado, reconociendo así la necesidad de establecer lineamientos que garanticen la estabilidad laboral del colaborador (2) de acuerdo a los hallazgos es imperante en el marketing interno determinar políticas de salario equitativas, las cuales deben instituirse y ajustarse de acuerdo a las labores desempeñadas y formación profesional de cada empleado; (3) en lo que respecta a la comunicación interna de la empresa es demandante que se ejecuten estrategias donde el proceso interactivo ocurra de forma bidireccional, a fin de hacer partícipe al colaborador de los cambios generados en la organización y en este sentido afianzar su sentido de pertenencia.

\section{REFERENCIAS}

Acopi, Encuesta de desarrollo empresarial: Barranquilla, Colombia (2017)

Araque, D. L.; J. M. Sánchez y A. F. Uribe, Relación entre marketing interno y compromiso organizacional en Centros de Desarrollo Tecnológico colombianos, doi.org/10.1016/j.estger.2016.12.005, Estudios Gerenciales, 33(142), 95-101 (2017)

Arias, F., El Proyecto de investigación: Introducción a la Metodologia cientifica, $6^{\circ}$ Ed., 31-34. Editorial Episteme, Caracas, Venezuela (2012)

Azêdo, D. F. y M. H. Alves, Práticas de marketing interno nas organizações de saúde e a sua influência na motivação dos colaboradores, doi: 10.1016/j.rpsp.2013.05.003, Revista Portuguesa de Saúde Pública, 31(2), 181-192 (2013)

Balemba, E. y D. Bugandwa, Internal marketing, employee job satisfaction, and perceived organizational performance in microfinance institutions, doi: 10.1108/IJBM-06-2015-0083, International Journal of Bank Marketing, 34(5), 773-796 (2016)

Bang W. S.; J. I. Shin y K.H. Chung, The source of competitive advantage: Internal Marketing, ISSN 1343-4500, Information, 20(4), 2663-2670 (2017)

Bansal, H.; M. Mendelson y B. Sharma, The impact of internal marketing activities on external marketing outcomes, doi: 10.1016/S1084-8568(01)00029-3, Journal of quality management, 6 (1), $61-76$ (2001)

Barra, A. M., Dirección Estratégica en las Superintendencias Del Estado de Chile, doi: 10.4067/S071807642017000300014, Información Tecnológica, 8(3), 129-138 (2017)

Berry, L. L., The employee as customer, Journal of retailing Banking, 3(1), 33-40 (1981)

Bohnenberger, M. C.; S. Schmidt; C. Damacena y F. J. Batle-Lorente, Internal marketing: a model for implementation and development, doi: 10.15665/dem.v17i1.1657, Dimension Empresarial, 17(1), 7-22 (2019)

Cámara de Comercio de Barranquilla y Fundesarrollo, Identificación y Caracterización de las necesidades Del Capital Humano actuales y con prospectiva para el Clúster Salud- Farma del Departamento del Atlántico, Colombia (2015)

Caridad, M.; C. Salazar y M. I. Castellano, Endomarketing: estrategia dinamizadora para la responsabilidad social del sector universitario, ISSN 0798 1015, Revista Espacios, 38(1), 6 -21, (2017)

Castillo, A. E.; G. Velandia; P. Hernández y W. Archibold, Gestión del conocimiento e innovación en las PYME exportadoras del sector industrial en Colombia, ISSN 0798 1015, Espacios, 38(34), 24-37 (2017)

Fang, S. R.; E. Chang; C. C. Ou y C .H. Chou, Internal market orientation, market capabilities and learning orientation, doi: 10.1108/EJM-06-2010-0353, European Journal of Marketing, 48(1), 170-192 (2014)

Fundesarrollo, La competitividad en Colombia y el departamento del Atlántico, Colombia (2017)

Gronross, C., Relatioship approach to marketing in service contexts: The marketing and organizational behavior Interface, doi: 10.1016/0148-2963(90)90037-E, Journal of Business, 20(1), 3-11 (1990)

Hernández, R.; C. Fernández y P. Baptista, Metodología de la investigación, 6 Ed., 001-607. Editorial McGraw-Hill México (2014)

Johnston R., The Customer as Employee, doi: 10.1108/EUM0000000001240, International Journal of Operations \& Production Management, 9(5), 15-23 (1989)

Fortenberry, J. L. y P. J. McGoldrick, Internal marketing: A pathway for healthcare facilities to improve the patient experience, doi: 10.1179/2047971915Y.0000000014, International Journal of Healthcare Management, 9(1), 28-33 (2016)

Jungsun, K.; J. Hak y K. Choong, Effects of corporate social responsibility and internal marketing commitment and turnover intentions, doi: 10.1016/j.ijhm.2016.02.007, International Journal of Hospitality Management, 55(1), 25-32 (2016) 
Kotler, P. y K. Keller, Dirección de Marketing, 12 Ed., 20-22 Pearson Educación, México (2006)

Lopez, A. F.; W. Sidele; C. Anderson y L. Sartor, Comunicação interna como ferramenta de endomarketing para maximização na competitividade: um estudo em pequenas empresas da cidade de Paranaguá no Brasil, doi: 10.3232/GCG.2016.V10.N2.03, Journal of public policy and marketing, 10(2), 54-70 (2016)

Mendoza, J.; M. Hernández y C. Tabernero, Retos y oportunidades de la investigación en marketing interno, ISSN: 1315-9518, Revista de Ciencias Sociales, 17(1), 110-125 (2011)

Ministerio de salud, Glosario, Colombia (2019)

Mohamed, R. y A. Pervaiz, Advances in the internal marketing concept: definition, synthesis and extension, doi: 10.1108/08876040010347589, Journal of Services Marketing, 14(6), 449-462 (2000)

Dantas, M. I. y A. M. Soares, Impacto do Marketing Interno sobre a Orientação para o Mercado em Empresas Brasileiras doi: 10.1590/1982-7849rac2016140069, Revista de Administração Contemporânea, 20(2), 197-215 (2016)

OCDE, C. C., Perspectivas económicas de América Latina 2017: Juventud, competencias y emprendimiento, doi: 10.1787/leo-2017-es, OECD Publishing, Paris (2017)

Papasolomou, I. y D. Vrontis, Building corporate branding through internal marketing: the case of the UK retail bank industry, doi: 10.1108/10610420610650864, Journal of Product \& Brand Management, 15(1), 33-47 (2006)

Payares, K. M.; A. Berdugo; M. C. Faria y E. Navarro, Endomarketing y calidad de servicio interno en las pequeñas y medianas empresas del sector salud, ISSN: 0798 1015, Revista Espacios, 38(57), 32-41 (2017)

Prieto, R.; C. Burgos; J. García y Y. Rincón, Mercadeo interno para optimizar la calidad de servicio en la banca universal. Revista Venezolana de Gerencia, ISSN: 1315-9984, Revista Venezolana de Gerencia, 21(73), 102-119 (2016)

Robledo, J. L. y A. M. Vallespín, Empirical analysis of the constituent factors of internal marketing orientation at Spanish hotels, ISSN: 2182-8458. Tourism \& Management Studies, 10(Especial), 151-157 (2014)

Rodrigues, A. Q. y C. Pires, A influência do marketing interno nas atitudes e comportamentos dos colaboradores: aplicação a uma organização de cuidados sociais e de saúde, doi: 10.1016/j.rpsp.2016.08.001 Revista Portuguesa de Saúde Pública, 34(3), 292-304 (2016)

Salehzadeh, R.; H. Jamkhaneh y S. Doosti, The effects of internal marketing mix on performance in a healthcare context, doi: 10.1504/IJBIR.2019.097251, International Journal of Business Innovation and Research, 18(2), 167-186 (2019)

Vieira-dos Santos, J. y G. Gonçalves, Organizational culture, internal marketing, and perceived organizational support in Portuguese higher education institutions, doi: 10.5093/jwop2018a5, Revista de psicologia del trabajo y de las organizaciones, 34(1), 38-41 (2018) 